\title{
Rho family GTPase-dependent immunity in plants and animals
}

\author{
Yoji Kawano ${ }^{1}$, Takako Kaneko-Kawano ${ }^{2}$ and Ko Shimamoto ${ }^{1+}$ \\ ${ }^{1}$ Laboratory of Plant Molecular Genetics, Nara Institute of Science and Technology, Ikoma, Japan \\ ${ }^{2}$ College of Pharmaceutical Sciences, Ritsumeikan University, Kusatsu, Japan
}

Edited by:

Shin-ichi Arimura, The University of

Tokyo, Japan

Reviewed by:

Ralph Hückelhoven, Technische Universitaet Muenchen, Germany Yan Zhang, Shandong Agricultural University, China

*Correspondence:

Yoji Kawano, Laboratory of Plant Molecular Genetics, Nara Institute of Science and Technology, 8916-5 Takayama, Ikoma,

Nara 630-0101, Japan

e-mail: y-kawano@bs.naist.jp

${ }^{\dagger}$ Dedicated to Ko Shimamoto, who

passed away on September 28, 2013.
In plants, sophisticated forms of immune systems have developed to cope with a variety of pathogens. Accumulating evidence indicates that Rac (also known as Rop), a member of the Rho family of small GTPases, is a key regulator of immunity in plants and animals. Like other small GTPases, Rac/Rop GTPases function as a molecular switch downstream of immune receptors by cycling between GDP-bound inactive and GTP-bound active forms in cells. Rac/Rop GTPases trigger various immune responses, thereby resulting in enhanced disease resistance to pathogens. In this review, we highlight recent studies that have contributed to our current understanding of the Rac/Rop family GTPases and the upstream and downstream proteins involved in plant immunity. We also compare the features of effector-triggered immunity between plants and animals, and discuss the in vivo monitoring of Rac/Rop activation.

\section{Keywords: Rac/Rop, small GTPase, plant immunity, PAMPs-triggered immunity, effector-triggered immunity}

\section{INTRODUCTION}

Recent studies on plant-pathogen interactions have revealed that plants have developed a two-branched system of immunity to prevent the invasion of pathogens. The perception of pathogen/microbe-associated molecular patterns (PAMPs/ MAMPs) by host pattern recognition receptors (PRRs) is important for the initiation step of innate immunity (Jones and Dangl, 2006; Dodds and Rathjen, 2010; Monaghan and Zipfel, 2012). In plants, host perception of PAMPs activates rapid defense responses such as the production of reactive oxygen species (ROS), induction of defense-related genes, and MAPK activation, designated PAMP-triggered immunity (PTI). Most PRRs are subdivided into three categories that include receptor-like kinases (RLKs), receptor-like proteins (RLPs), and receptor-like cytoplasmic kinases (RLCK; Monaghan and Zipfel, 2012). RLKs consist of an extracellular domain, a transmembrane domain, and an intracellular kinase domain, whereas RLPs lack the intracellular kinase domain and RLCKs only possess a cytoplasmic kinase domain. Structural studies show that RLKs perceive signals through their extracellular domains and transmit signals through their intracellular signaling molecules using their kinase domains. The Arabidopsis and rice genomes encode more than 600 and $1100 \mathrm{RLKs} / \mathrm{RLCKs}$, respectively, that participate in various cellular signaling processes and developmental events (Shiu et al., 2004). Chitin, found in pathogenic and non-pathogenic fungi, is one of the best-characterized PAMPs (Gust et al., 2012). Two lysine motif (LysM)-containing PRRs, OsCEBiP, and OsCERK1, play a vital role in chitin signaling (Kaku et al., 2006; Shimizu et al., 2010). OsCEBiP is an RLP that lacks an intracellular kinase domain and directly binds chitin, whereas OsCERK1 is an RLK and does not directly bind chitin. These two immune proteins form a receptor complex that transduces the chitin signals to downstream components (Kaku et al., 2006; Shimizu et al., 2010; Shinya et al., 2012).

To promote pathogen virulence, pathogen effectors target plant proteins and, thus, perturb host cell physiology and immunity. As a result, a second line of plant defense counterattacks the pathogens. This defense system is termed effector-triggered immunity (ETI; Jones and Dangl, 2006; Dodds and Rathjen, 2010). Compared to PTI, the ETI response is often more robust and faster. Disease resistance $(\mathrm{R})$ proteins act as intracellular receptors for the direct or indirect recognition of specific pathogen effectors [also called avirulence (Avr) proteins]. ETI triggered by R proteins results in strong host responses accompanied by cell death. Most $\mathrm{R}$ genes encode members of the nucleotide-binding (NB) domain and leucinerich repeat (LRR) domain (NLR, also called NB-LRR) family that often display a tripartite domain architecture and are subdivided according to their $\mathrm{N}$-terminal domains into coiled-coil (CC-NLR) and Toll/Interleukin-1 Receptor homology (TIR-NLR) subclasses. Although sharing broad structural similarities, many NLR proteins also show unique structural variations that are important for their function and subcellular localization (Lukasik and Takken, 2009).

The Rho family of GTPases belongs to the Ras superfamily of small GTPases. The Rho family in animals is further divided into 3 subfamilies: the Rho, Rac, and Cdc42 proteins. In contrast, the Rho family in plants converges into a single subfamily that is distinct from all major subfamilies of animal Rho-GTPases (Li et al., 1998). The plant Rho subfamily is most closely related to the animal Rac subfamily (about 65\% identity at the amino acid level). Thus, these plant GTPases are called Rac-like (Rac family) or Rop-like (Rho-related GTPases of plants) proteins (Kawasaki et al., 1999; Winge et al., 2000). The Rac/Rop family is one of the most important regulators of signal transduction in plants, participating in pathways that influence 
growth and development and the adaptation of plants to various environmental situations (Berken, 2006). Evidence is accumulating that the Rac/Rop family plays a critical role in plant immunity (Kawano et al., 2010b; Kawano and Shimamoto, 2013). Among the Rac/Rop family of small GTPases in plants, we will highlight two small GTPases in the Rac/Rop family, rice Oryza sativa Rac1 (OsRac1) and barley Hordeum vulgare RacB (HvRacB), in this review.

\section{Rac/Rop FAMILY OF SMALL GTPases IN PLANTS}

Proteins in the Rac/Rop GTPase family contain five highly conserved G-boxes (G1-G5; Figure 1; Paduch et al., 2001; Wennerberg et al., 2005). G1, G3, G4, and G5 play critical roles in binding to GTP/GDP and hydrolysing GTP to GDP. The G2 box is known to be the effector domain that is essential for binding to downstream effector proteins. The C-terminal polybasic region and post-translational modification site play important roles in subcellular localization and small GTPase function. Rac/Rop GTPases can be divided into two types based on their C-terminal motifs (Winge etal., 2000). Type I Rac/Rop GTPases possess a conserved CaaL (a: aliphatic amino acid) motif, whereas type II proteins lack this motif but retain a cysteine-containing element for membrane anchoring. All type-I Rac/Rop family members are putatively prenylated; the type-II proteins are palmitoylated but not prenylated (Lavy et al., 2002).

There are seven Rac/Rop family genes in rice (Miki et al., 2005), 6 genes in barley (Schultheiss et al., 2003), and 11 genes in Arabidopsis (Winge et al., 2000). All seven members of the Rac/Rop family in rice are expressed in seedlings, leaf sheaths, stems, and roots, but expression of OsRac2, 6, and 7 is much lower than that in leaf blades (Chen et al., 2010b). The expression level of OsRac7 is also low in panicles, immature seeds, and cultured cells. These different tissue specificities suggest distinct roles for different Rac/Rop small GTPases in the various signaling pathways in rice. OsRac5-7 are type I Rac/Rop proteins that have a conserved CaaL motif at the C-terminus, and OsRac1-4 are type II Rac/Rop proteins that possess a truncated but functional post-translational modification motif (Chen et al., 2010b). In general, members of the Rac/Rop family are localized mainly at the plasma membrane, but some signals are detected in the cytoplasm and the nucleus
(Chen et al., 2010b). Members of the rice type I Rac/Rop family are more often localized in the nuclei and the cytoplasm than the type II proteins. Constitutively active (CA) forms of OsRac/Rops tend to show plasma membrane localization more often than their dominant negative (DN) forms. OsRac3 and OsRac4 have the highest percentage of plasma membrane localization among the rice Rac/Rop GTPases.

\section{REGULATORS OF Rac/Rop GTPases}

The ratio between the GDP-bound inactive and GTP-bound active forms of Rac/Rop depends on the activity of regulating proteins (Figure 2). GTPase-activating protein (GAP) works as a negative regulator by promoting the intrinsic GTPase activity of Rac/Rop and reconverting it to the inactive GDP - Rac/Rop. Guanine nucleotide dissociation inhibitor (GDI) inhibits the exchange of GDP for GTP. Guanine nucleotide exchange factor (GEF) enhances the release of GDP from Rac/Rop, thereby promoting the binding of GTP. GTP-Rac/Rop interacts with downstream effectors and then triggers various cellular responses.

More than 30 RacGEFs have been described in animals; most of them share conserved Dbl homology (DH) and pleckstrin homology (PH) domains (Bos et al., 2007). Notably, only two DH-PH RacGEFs with significant similarity to human SWAP70 have been recently found in plants (Shinohara et al., 2002; Yamaguchi et al., 2012). SWAP70 contains both $\mathrm{DH}$ and $\mathrm{PH}$ domains, but their arrangement is the reverse of that in typical DH-PH-type Rho GEFs, wherein the $\mathrm{DH}$ domain is flanked by a C-terminal $\mathrm{PH}$ domain. In addition, plants possess a unique family of RacGEFs whose members specifically activate Rac/Rop GTPases in vitro (Berken et al., 2005). RacGEFs are characterized by a highly conserved catalytic domain called a plant-specific Rop nucleotide exchanger (PRONE). PRONE was found to promote nucleotide dissociation from Rac/Rop with catalytic properties comparable to DH-PH GEFs. Based on the three-dimensional structure of PRONE GEF, catalysis follows a push-and-pull mechanism affecting the switch regions of small GTPases (Thomas et al., 2007).

\section{Rac/Rop IN PTI}

Constitutively active form (CA)-OsRacl causes hypersensitive response (HR)-like responses and greatly reduces disease lesions

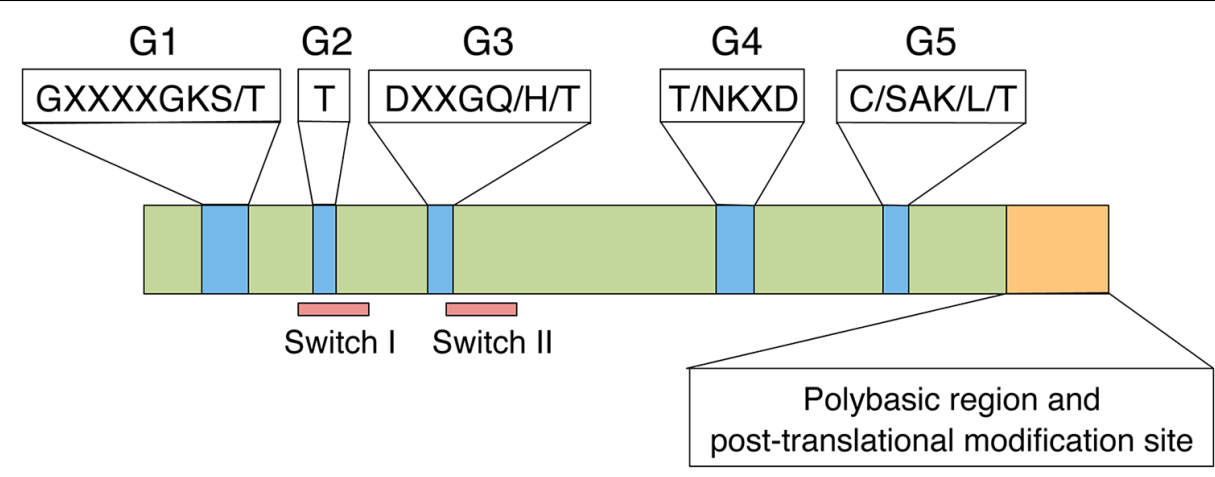

FIGURE 1 | Functional domains of the Rac/Rop family. The Rac/Rop family shares a set of five conserved G boxes (Blue), a polybasic region and a post-translational modification site (Orange). 


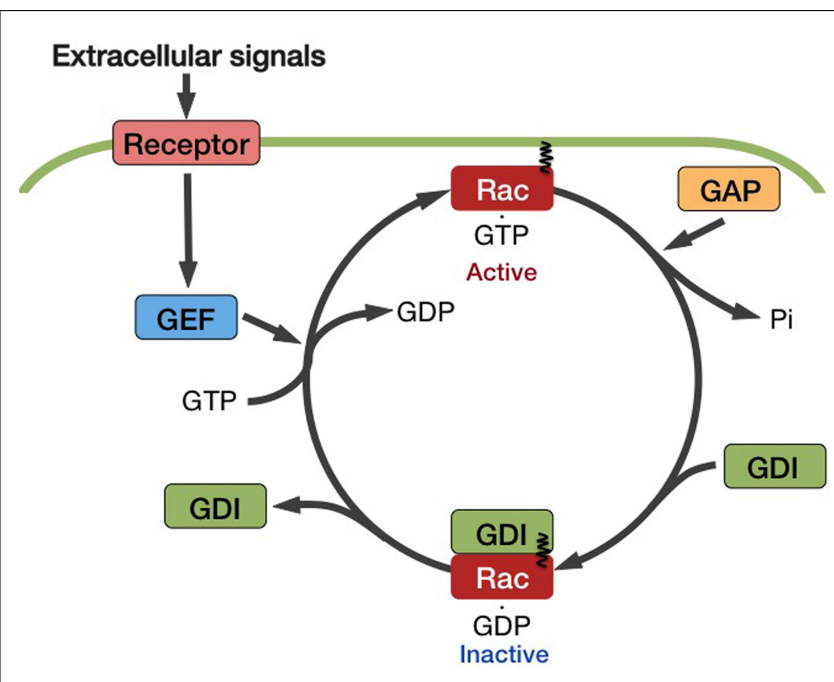

FIGURE 2 | Mode of activation of the Rac/Rop family GTPases. The ratio of these two forms of Rac/Rop is dependent of the activity of regulating factors. GTPase-activating proteins (GAPs) act as negative regulators by accelerating the intrinsic GTPase activity of Rac/Rop and reconverting it to the inactive GDP. Rac/Rop. Guanine nucleotide dissociation inhibitors (GDIs) inhibit the exchange of GDP for GTP. Guanine nucleotide exchange factors (GEFs) facilitate the release of GDP from Rac/Rop, thereby promoting the binding of GTP.

induced by virulent races of the rice blast fungus (Kawasaki et al., 1999; Ono et al., 2001). CA-OsRac1 also causes resistance against virulent races of bacterial blight, enhances production of phytoalexins, and alters expression of defense-related genes. These results indicate that OsRac1 acts as a positive regulator of PTI in rice. To further elucidate the roles of all seven Rac/Rop family proteins in rice immunity, we made knockdown plants of each OsRac family gene and performed the infection assays using a virulent race of rice blast fungus (Chen et al., 2010b). OsRac4 and OsRac5 RNAi plants have shorter lesions caused by a virulent race of rice blast fungus, suggesting that OsRac4 and OsRac5 are negative regulators of PTI. There are no obvious effects in OsRac3, OsRac6, and OsRac7 RNAi plants on the lesions induced by a virulent race of rice blast fungus. OsRac6 plays a modest role in defense; however, based on an overexpression study, OsRac6, also known as $O s R a c B$, was proposed to be a negative regulator in defense (Jung et al., 2006). The existence of positive and negative roles for Rac/Rop GTPases in rice innate immunity also indicates the complexity of Rac/Rop functions in disease resistance.

\section{Rac/Rop AND IMMUNE RECEPTORS IN PTI}

Studies in other research areas provide clues for how PRRs activate Rac/Rop family GTPases in plant immunity. The Rac/Rop family is implicated in signaling downstream of RLK CLAVATA1, a receptor regulating the balance between cell differentiation and cell division in aerial meristems (Trotochaud etal., 1999). An unidentified Rac/Rop GTPase was found in the immunoprecipitate of the $450 \mathrm{kDa}$ active CLAVATA1 complex. Moreover, McCormick and colleagues found that PRONE-type AtRopGEF associates with pollen-specific RLKs,
LePRK1, and LePRK2 (Kaothien et al., 2005). They characterized AtPRK2a, an Arabidopsis homolog of LePRK2, and verified the physical interaction between AtPRK2a and the Arabidopsis PRONE-type AtRopGEF12, implying that RopGEF activity is regulated by RLKs. Phosphorylation of the C-terminus of AtRopGEF12 appears to be important for regulating GEF activity (Zhang and McCormick, 2007). A phospho-mimicking mutation at a highly conserved serine residue in the Cterminus of AtRopGEF12 results in the loss of the C-terminal autoinhibition.

Oryza sativa Racl has been shown to participate in PTI induced by elicitors derived from fungal pathogens such as chitin and sphingolipids (Ono etal., 2001; Suharsono etal., 2002). How the OsCEBiP-OsCERK1 chitin-receptor complex transmits extracellular chitin signals to downstream components was unclear until recently. An OsCEBiP/OsCERK1-OsRacGEF1OsRac1 module participates in the immunoresponse caused by chitin, and chitin activates OsRac1 within $3 \mathrm{~min}$ of chitin treatment (Figure 3; Akamatsu et al., 2013). OsRacl interacts with a PRONE-type GEF called OsRacGEF1 that is directly phosphorylated at Ser549 after chitin treatment by OsCERK1. This phosphorylation leads to the activation of OsRacGEF1, resulting in the activation of OsRac1. Knockdown of OsRacGEF1 compromises the expression of defense-related genes and attenuates disease resistance to virulent races of rice blast fungus. Overall, these results support the hypothesis that OsRacGEF1 is a direct substrate of OsCERK1 chitin receptor and that OsRacGEF1phosphorylated by OsCERK1 leads to OsRac1 activation. A GEF protein, SWAP70, also interacts with OsRacl and the DH-domain exhibited GEF activity toward OsRacl in vitro, indicating that plants possess a functional DH domain (Yamaguchi et al., 2012). In addition, OsSWAP70 regulates chitin-induced ROS production and defense gene expression in rice. Thus, it is likely that SWAP70 functions as a GEF for OsRac1 in rice.

AtROP6 orchestrates developmental and disease resistance signaling (Poraty-Gavra et al., 2013). AtROP6 expression is induced by the plant hormone auxin and is detected in the root meristem, lateral root initials, and leaf hydathodes. The expression of DN-AtRop6 induces small, multiple inflorescence stems, twisted leaves, deformed leaf epidermis pavement cells, and differentially organized cytoskeletons. The expression of DN-AtRop6 leads to major changes in gene expression for proteins participating in constitutive salicylic acid (SA)-mediated defense responses. Accordingly, the free and total SA levels in DN-AtRop6 without an infection resemble those of wild-type plants inoculated with a virulent powdery mildew pathogen. The constitutive SA responses in DN-AtRop6 are suppressed in mutants defective in SA signaling [non-expressors of PR gene1 (npr1)] or biosynthesis [SA induction deficient2 (sid2)]. However, the DN-AtRop6 $n$ pr1 and DN-AtRop6 sid2 double mutants retain the aberrant developmental phenotypes, implying that AtRop6's function in development is not related to the constitutive SA response. $D N$ AtRop6 plants exhibit the developmental phenotype of enhanced pre-invasive defense responses to a host-adapted virulent powdery mildew fungus, Golovinomyces orontii, but are impaired in pre-invasive defenses upon inoculation with a non-adapted 


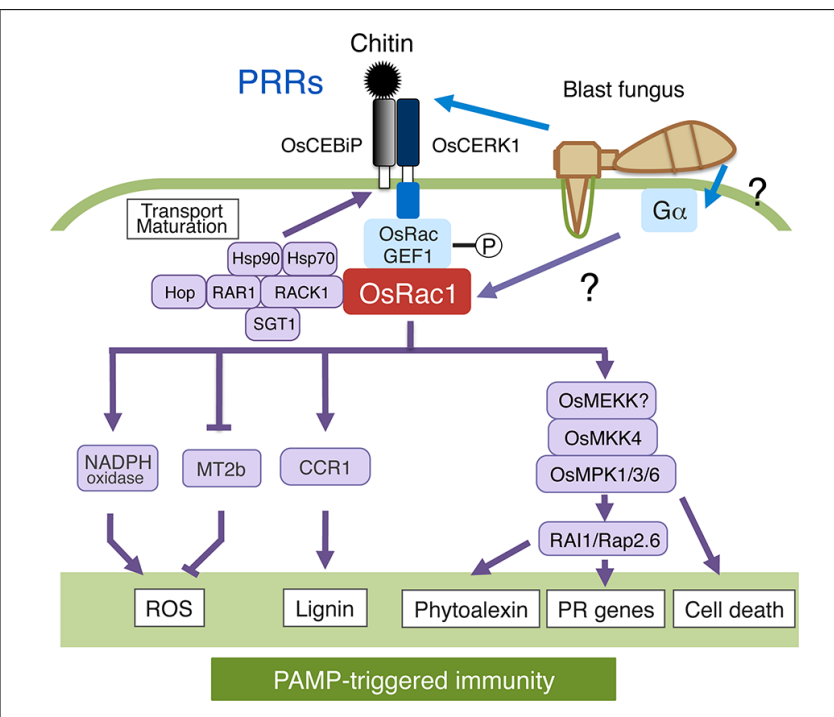

FIGURE 3 | Roles of OsRac1 in PAMP-triggered immunity (PTI). OsCERK1-dependent phosphorylation of OsRacGEF1 leads to the activation of OsRac1. OsRac1 activates OsMPK1/3/6 through OsMEKK4; activated OsMPK3/6 activates the transcription factor RAl1 as well as Rap2.6. The chaperone and co-chaperone complex of Hop/Sti1-Hsp90 facilitates the maturation and transport of OsCERK1. OsRac1 plays a dual role as an activator of NADPH oxidase and a suppressor of reactive oxygen species (ROS) scavenging. Lignin: cell wall, Phytoalexin: antibacterial substance, PR genes: Pathogenesis-related genes.

powdery mildew, Blumeria graminis f. sp. hordei (Bgh). The host-adapted powdery mildew, G. orontii, has a reduced reproductive fitness on DN-AtRop6 plants, a phenotype that is retained in mutants defective in SA biosynthesis or signaling. These results indicate that both the morphological aberrations and the enhanced disease resistance effect to host-adapted G. orontii in DN-AtRop6 are independent of SA-dependent defense signaling.

\section{Rac/Rop AND NADPH OXIDASE IN PTI}

In plants, ROS can strengthen host cell walls via glycoprotein cross-linking or cause lipid peroxidation and membrane damage. However, ROS are one of the most important second messengers in plant defense (Torres and Dangl, 2005). Additional regulatory functions for ROS in defense occur in conjunction with other plant signaling molecules, particularly SA and nitric oxide. Cotton (Gossypium hirsutum) Rac13 triggers the production of ROS, which may serve as a signal for secondary wall formation in cotton (Potikha et al., 1999). The CA mutant of barley HvRacl promotes ROS accumulation in infected leaves (Pathuri et al., 2008); however, this effect is observed only in cells where a fungal attempt to penetrate failed, i.e., where neither a fungal haustorium initial nor an elongated secondary fungal hyphae developed. In contrast, there are no detectable effects on ROS production from the expression of two other HvRacs, $C A-H v R a c B$, and $C A-H v R a c 3$. Rop guanosine triphosphatase activating protein 4 (RopGAP4) in Arabidopsis is involved in the generation of ROS required for responses to oxygen deprivation (Baxter-Burrell et al., 2002).
Oryza sativa Rac1 is a regulator of ROS production and cell death in rice (Kawasaki et al., 1999). CA-OsRacl enhances PAMPsinduced ROS production and resistance to pathogens in rice (Figure 3; Kawasaki et al., 1999; Ono et al., 2001). The direct interaction between OsRacl and the N-terminal region of NADPH oxidase [also called Respiratory burst oxidase homologs (Rboh)], including the two EF-hand motifs which is the most common calcium-binding motif, is required for the activation of Rboh by OsRac1 (Wong et al., 2007). The cytosolic $\mathrm{Ca}^{2+}$ concentration may modulate NADPH oxidase activity by regulating the direct interaction between OsRacl and OsRboh. Structural analyses further support the hypothesis of a direct interaction between OsRacl and RbohB (Oda et al., 2010; Kosami et al., 2014). The OsRacl binding interface in OsRbohB is located in the flanking region of the coiled-coil region at the $\mathrm{N}$-terminus. The structure of this binding region is not similar to those previously identified as Rac binding motifs in animals. Thus, OsRac1 binds to OsRbohB in a manner distinct from known interactions between Rac and its target proteins. The expression of Metallothionein $2 b$ (MT2b), a ROS scavenging gene, is synergistically down-regulated by OsRacl and rice blast-derived elicitors (Wong et al., 2004). Collectively, OsRac1 might play a dual role as an inducer of ROS production and a suppressor of ROS scavenging.

\section{Rac/Rop AND MAPK SIGNALING IN PTI}

MAPK signaling in all eukaryotes is organized in three-tiered modules comprising a MAPK kinase kinase (MAPKKK/MEKK), a dual-specificity MAPKK (MKK), and a MAPK (MPK), within which phosphorylation signals are transduced linearly from the MAPKKK to the MAPK (Rodriguez et al., 2010; Samajova et al., 2013). The CA MKK NtMEK2 activates two MPKs, NtSIPK, and NtWIPK, followed by an induced HR and defense gene expression (Yang et al., 2001). A complete MPK cascade involving MEKK1MKK4/MKK5-MPK3/MPK6 has been previously reported in Arabidopsis (Asai et al., 2002). OsMPK1 protein levels are strongly reduced in OsRac1-knockdown cells and in the trimeric G-protein $\alpha$ subunit mutant $d 1$, and sphingolipid elicitor-induced OsMPK1 activation is greatly reduced in both mutant cells (Figure 3; Lieberherr et al., 2005). These results suggest that these two GTP-binding proteins contribute to the stability of OsMPK1 protein and, possibly, for its activation as well. In animals, Ras-like GTPases are involved in the upstream signaling for MAPK cascade activation. These Ras-MAPK or G-protein-MAPK cascades occur in response to various stimuli, such as hormones or environmental stresses. G $\alpha$ functions upstream of OsRac1 in the sphingolipid elicitor signaling pathway, leading to the induction of ROS production and defense gene expression (Suharsono etal., 2002). Therefore, a MAPK cascade may be located downstream of these two G-proteins along with other pathways. In fact, our immunoprecipitation assay showed that OsMPK1 and OsRac1 form the same protein complex, indicating that OsRacl activates OsMPK1 in a manner similar to the Ras-MAPK pathway in animals. The mechanism of how $\mathrm{G} \alpha$ activates OsRac1 in plants remains to be studied.

The basic helix-loop-helix transcription factor Rac Immunity 1 (RAI1) is up-regulated 15-30 min after chitin treatment (Figure 3; 
Kim et al., 2012). Moreover, the expression of CA-OsRacl upregulates RAI1 expression in rice suspension cells. Accordingly, RAI1 T-DNA activation-tagged lines show enhanced resistance to a virulent race of blast fungus, implying that RAI1 is a positive regulator of plant immunity and is involved in the OsRac1-dependent chitin pathway. A microarray analysis of cells transformed with an inducible RAI1 construct showed increased gene expression of PAL1 and the transcription factor OsWRKY19 after induction, suggesting that these genes are regulated by RAI1. Chitin elicitor activates Oryza sativa MAPK kinase 4 (OsMKK4) as well as two MAPKs, OsMPK3, and OsMPK6 (Kishi-Kaboshi et al., 2010). OsMKK4-dependent phosphorylation of OsMPK3 and OsMPK6 appears to be essential for the chitin elicitor-induced biosynthesis of diterpenoid phytoalexins that act as toxins to restrict Magnaporthe oryzae infection. OsMAPK3 and OsRac1 form the same complex as previously reported for OsMPK1 (Lieberherr etal., 2005; Kim et al., 2012). The expression levels of the two downstream genes, PAL1 and OsWRKY19, are increased by overexpression of OsMPK6 and/or OsMPK3 together with the active form of OsMKK4. Moreover, RAI1, a transcription factor, is directly phosphorylated by OsMPK3/6 in an active form of OsMKK4-dependent manner in vitro. Taken together, our results indicate that RAI1 is regulated by OsRacl through an OsMPK3/6 cascade. Similarly, a different type of transcription factor OsRap2.6 may be controlled by OsRac1 through an OsMPK3/6 pathway (Wamaitha et al., 2012).

\section{Rac/Rop AND DOWNSTREAM PROTEINS IN PTI}

Oryza sativa Racl appears to form a "defensome network" consisting of various proteins that collectively regulate rice immunity (Figure 3; Kawano etal., 2010b; Kawano and Shimamoto, 2013). This network might include chitin receptor OsCERK1 as well as OsRacGEF1, the heat shock protein 90 (Hsp90), Hsp70, co-chaperone Hop/Sti1, the scaffold protein RACK1, the lignin biosynthesis enzyme O. sativa CinnamoylCoA reductase 1 (OsCCR1), OsMPK3, OsMPK6, and RAI1 (Lieberherr et al., 2005; Thao et al., 2007; Nakashima et al., 2008; Chen etal., 2010a; Kim etal., 2012; Akamatsu etal., 2013). Hop/Stila and Hsp90 directly interact with OsCERK1 (Chen et al., 2010a). Co-chaperone Hop/Stil is transported from the endoplasmic reticulum (ER) to the plasma membrane, and the Hop/Stil-Hsp90 chaperone complex contributes to the maturation and intracellular transport of the OsCERK1 complex (Chen et al., 2010a; Akamatsu et al., 2013). In fact, the knockdown of Hop/Stil suppresses chitin-triggered pathogenesis-related gene expression and disease resistance to virulent races of rice blast fungus. The transport of the OsCERK1 complex is mediated by the small GTPase Sar1 that regulates ER-to-Golgi trafficking because overexpression of CA-Sarl compromised the transport of OsCERK1 from the ER to the plasma membrane (Chen et al., 2010a). These results suggest that the Hop/Sti1-HSP90 chaperone complex plays an important role in the maturation and transport of PRRs and may function to link PRRs and Rac/Rop GTPases.

RACK1 associates with many signaling proteins in animals and acts as a scaffolding protein in a number of signaling pathways (McCahill et al., 2002). Although RAR1, Hsp90, and Hsp70 are present in the OsRacl complex, none of them appear to interact directly with OsRac1 (Figure 3; Thao et al., 2007). OsRac1 appears to form a complex with these chaperones and co-chaperones through RACK1 because RACK1 directly interacts with OsRac1 as well as with SGT1, RAR1, and Hsp90 (Nakashima et al., 2008). The interaction of these three (co)-chaperones in rice seems to occur mainly in PTI (Thao et al., 2007; Wang et al., 2008). Accordingly, treatment with geldanamycin, an Hsp90 inhibitor, compromises OsRac1-HSP90 complex formation (Thao et al., 2007). RACK1 also plays a key role in the production of ROS and PTI (Nakashima etal., 2008). RACK1 is involved in hormone signaling and development in plants (Chen et al., 2006; Nakashima et al., 2008). OsRac1 positively regulates RACK1 at both the transcriptional and posttranscriptional levels. RACK1 transcription is also induced by chitin, a fungal elicitor, and by various plant hormones including abscisic acid, jasmonate, and auxin. RACK1 interacts with the N-terminus of NADPH oxidase, together with RAR1 and SGT1. Based on these results, two functions for RACK1 in rice innate immunity can be envisaged. One hypothesis is that RACK1 is a component of the defensome network consisting of OsRac1, RAR1, SGT1, Hsp90, and Hsp70 and functions as a scaffolding protein for this immune complex. Another possible function is that RACK1 is a component of the NADPH oxidase complex together with OsRac1 and regulates ROS production at an early stage in immune responses.

Lignin, a major component of secondary cell walls, is a heterogeneous tridimensional phenolic polymer resulting from the oxidative polymerization of monolignols (Boerjan et al., 2003). OsCCR1, an enzyme involved in lignin biosynthesis, is a target protein of OsRac1 (Figure 3; Kawasaki et al., 2006). Lignin is an important factor in plant defense responses because it forms an undegradable mechanical barrier to most pathogens. Sphingolipid treatment induces the expression of OsCCR1. OsRac1 binds OsCCR1 in a GTP-dependent manner, and the interaction of OsCCR1 with OsRac1 leads to the enzymatic activation of OsCCR1 in vitro. Suspension cells expressing CA-OsRac1 accumulate lignin through enhanced OsCCR1 activity and increased ROS production. Thus, OsRac1 likely controls lignin synthesis through regulation of both NADPH oxidase and OsCCR1 activities during defense responses.

\section{Rac/Rop IN ETI}

Oryza sativa Racl is involved in PTI as well as ETI including $P i-a$ and Pit-mediated disease resistance (Figure 4; Ono etal., 2001; Chen etal., 2010b; Kawano etal., 2010a,b; Kawano and Shimamoto, 2013). Pi- $a$ and Pit are the resistance genes to rice blast fungus. OsRacl interacts directly with the NB-ARC domain of Pit at the plasma membrane (Kawano et al., 2010a). OsRacl is activated by the active form of Pit at the plasma membrane and thereby induces ROS production as well as the HR. Recently, to decipher the mechanisms involved in the localization of Pit, we searched for consensus sequences in Pit that are associated with membrane localization and found a pair of potential palmitoylation sites in the $\mathrm{N}$-terminal coiled-coil region (Kawano et al., 2014). Although wild-type Pit is localized predominantly to the plasma membrane, this membrane localization was 


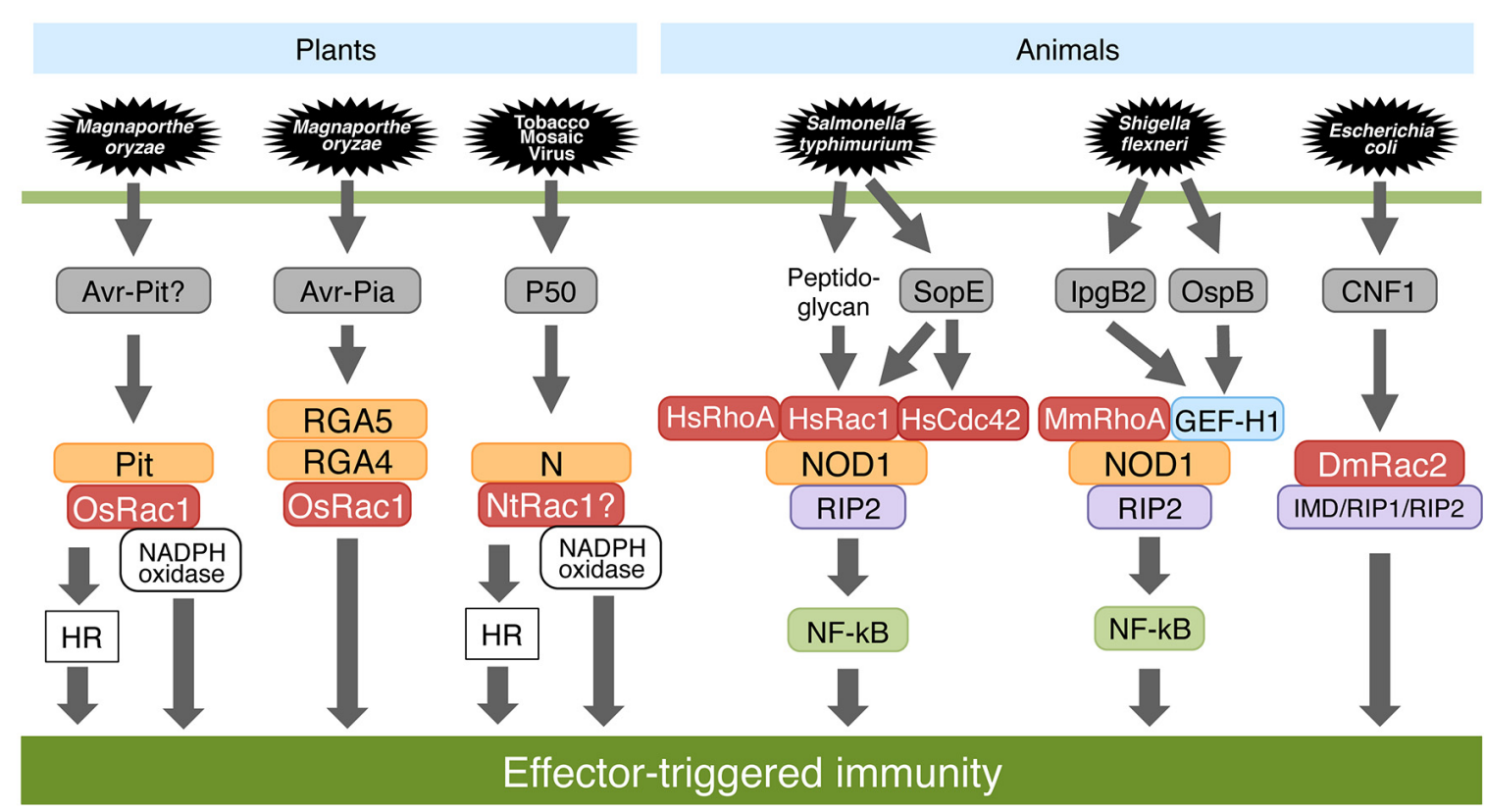

FIGURE 4 | Effector-triggered immunity (ETI) in plants and animals. OsRac1 contributes to Pit- and Pia-mediated ROS production as well as the hypersensitive response (HR) in rice and is required for disease resistance to avirulent races of blast fungus. Tobacco NtRac1 also acts as a downstream molecule of $\mathrm{N}$ resistance protein. In human and mouse, an NLR protein, NOD1, monitors the activation state of the Rho family GTPases that are targeted by virulence effectors caused by pathogenic microbes. In flies, an Escherichia coli-derived effector molecule CNF1 modifies DmRac2 to trigger an immune response. compromised in a Pit mutant in which a pair of cysteine residues that are potential palmitoylation sites were substituted with Alanines, indicating that palmitoylation is required for the plasma membrane localization of Pit. This palmitoylation-deficient Pit mutant has a significantly lower affinity for OsRacl on the plasma membrane, resulting in failed Pit-mediated cell death, ROS production, and disease resistance to rice blast fungus. These results indicate that palmitoylation-dependent membrane localization of Pit is required for the interaction with and the activation of OsRac1, and that OsRacl activation by Pit is vital for Pitmediated disease resistance to rice blast fungus. Terauchi and colleagues cloned Pia, resistance genes, and found that the Pia locus contains two NLR-type $R$ genes, $R G A 4$, and $R G A 5$, that are located next to each other in the genome and are oriented in opposite directions (Okuyama et al., 2011). RGA4 and RGA5 are a pair of $\mathrm{R}$ proteins and act together to trigger disease resistance against pathogens. Thus, the deletion of either of these $\mathrm{R}$ proteins fails to induce Pia-mediated resistance. RGA4 and RGA5 form a hetero-complex and interact through their coiledcoil domains. Recently, the different roles of RGA4 and RGA5 were resolved (Cesari et al., 2013; Césari et al., 2014). RGA5 directly interacts with and recognizes of the M. oryzae effector Avr-Pia as well as Avr1-CO39 and acts as a sensor for effector proteins. Interestingly, RGA4 and RGA5 have opposing functions: RGA4 constitutively induces immune responses, whereas RGA5 suppresses RGA4-induced immune responses. These findings raise interesting questions about how these two $\mathrm{R}$ proteins activate OsRac1. Moreover, overexpression of DN-OsRac1 in tobacco leaves suppresses the synchronous production of HR and ROS triggered by $N$ as well as Pto resistance genes (Moeder et al., 2005). Overall, it is likely that OsRacl generally functions downstream of several R proteins.

Many previous studies using inhibitors and agonists of heterotrimeric G-proteins in several plant species have suggested that G-proteins are involved in defense signaling. The $d 1$ mutant showed that $\mathrm{G} \alpha$ is involved in disease resistance (Suharsono et al., 2002). d1 mutants exhibit a highly reduced HR to infection by an avirulent race of rice blast fungus and enhanced hyphal extension, indicating that $\mathrm{G} \alpha$ is involved in $R$-genemediated disease resistance in rice. Activation of $P B Z 1$ expression, a pathogenesis-related gene, in $d 1$ with rice blast fungus is delayed for $24 \mathrm{~h}$ relative to the wild type (WT). G $\alpha$ expression is induced by an avirulent race of rice blast, and the expression of CA-OsRac1 in $d 1$ mutants restores sphingolipid elicitor-dependent $P B Z 1$ expression and disease resistance to an avirulent rice blast fungus. These results imply that the heterotrimeric G-protein functions upstream of OsRacl in the early steps of signaling. $\mathrm{G} \alpha$ is also involved in PBZ1 expressioninduced by the plant activator probenazole (Iwata et al., 2003). The expression of CA-OsRacl also induces the expression of PBZ1. Thus, OsRacl is probably activated by probenazole downstream of $\mathrm{G} \alpha$.

Phosphatidic acid (PA) is involved in numerous stress responses of plants. Intracellular PA levels increase under various biotic and abiotic stress conditions, including pathogen infection (Young et al., 1996; van der Luit etal., 2000); however, the physiological roles of PA in the stress response remain largely unclear. Treatment with PA induces cell death and elevates the levels of 
ROS in the leaves and single cells of Arabidopsis (Park et al., 2004). Arabidopsis leaves expressing a CA-AtRop2 develop earlier cell death and higher levels of ROS production than WT, whereas cell death in those expressing a $D N-A t R o p 2$ is later and ROS production is lower. However, in the absence of exogenous PA, spontaneous cell death or ROS induction does not occur in CA-AtRop2 plants, indicating that the activation of AtRop2 is required for ROS production but is not sufficient to induce the ROS generation pathway. These results suggest that PA regulates additional pathways required for active AtRop2dependent ROS production. Therefore, PA may be an important regulator of AtRop2-mediated ROS generation and the cell death process during various stress and defense responses in Arabidopsis.

At present, we do not know the mechanism by which OsRac1, a single Rho family GTPase, has highly diverse functions. Differences in tissue distribution in planta or subcellular distribution in cells might lead to the functional diversity observed in OsRac1, but further studies are necessary to resolve this issue.

\section{Rac/Rop AND THE CYTOSKELETON IN SUSCEPTIBILITY TO DISEASE}

Barley HvRacB contributes to the plant's susceptibility to barley powdery mildew (Schultheiss et al., 2002). The function of HvRacB is related to that of the major susceptibility factor MILDEW LOCUS O (MLO; Figure 5) and ROR1, another locus that is required for recessive mlo-specified resistance (Schultheiss et al., 2002, 2003). The expression of CA-HvRacB causes enhanced susceptibility to penetration and haustorium formation by the barley powdery mildew fungus $B g h$ and causes depolarized growth of root hairs (Schultheiss et al., 2005; Pathuri et al., 2008). By contrast, silencing of $H v R a c B$ by RNAi limits fungal success in haustorium formation and causes a failure of root hair outgrowth (Hoefle et al., 2011). The biotrophic powdery mildew fungus $B g h$ penetrates susceptible barley (Hordeum vulgare L.) by invading epidermal cells that remain intact during fungal development. The actin cytoskeleton is differentially reorganized in susceptible and resistant plants challenged by Bgh. Actin filaments are highly polarized toward the sites of attempted penetration of Bgh in resistant plants, whereas a more subtle reorganization takes place around fungal haustoria in susceptible plants (Opalski et al., 2005). Polarized distribution of the actin cytoskeleton toward sites of fungal attack is closely related to the successful prevention of fungal invasion. Moreover, overexpression of $C A-H v R a c B$ partly inhibits the polarized distribution of F-actin toward sites of $B g h$ invasion, whereas knockdown of $\mathrm{HvRacB}$ enhances actin focusing. Overall, $\mathrm{HvRacB}$ and $\mathrm{MLO}$ are host proteins involved in the modulation of the actin cytoskeleton at the interface between the host plant and Bgh. CA-HvRacB interacts with HvRIC171, a barley Cdc42/Rac interactive binding domain (CRIB)-motif protein. CAHvRacB and fungal attack promote recruitment of HvRIC171 to the cell periphery or sites of fungal entry, respectively, (Schultheiss et al., 2008). The overexpression of HvRIC171, similar to that of $C A-H v R A C B$, renders the plant more susceptible to invasion by $B g h$, whereas, expression of a 46-amino-acid HvRIC171-CRIB peptide, which is sufficient to interact with CA-HvRacB, has a DN effect and decreases susceptibility to Bgh. Taken together, these

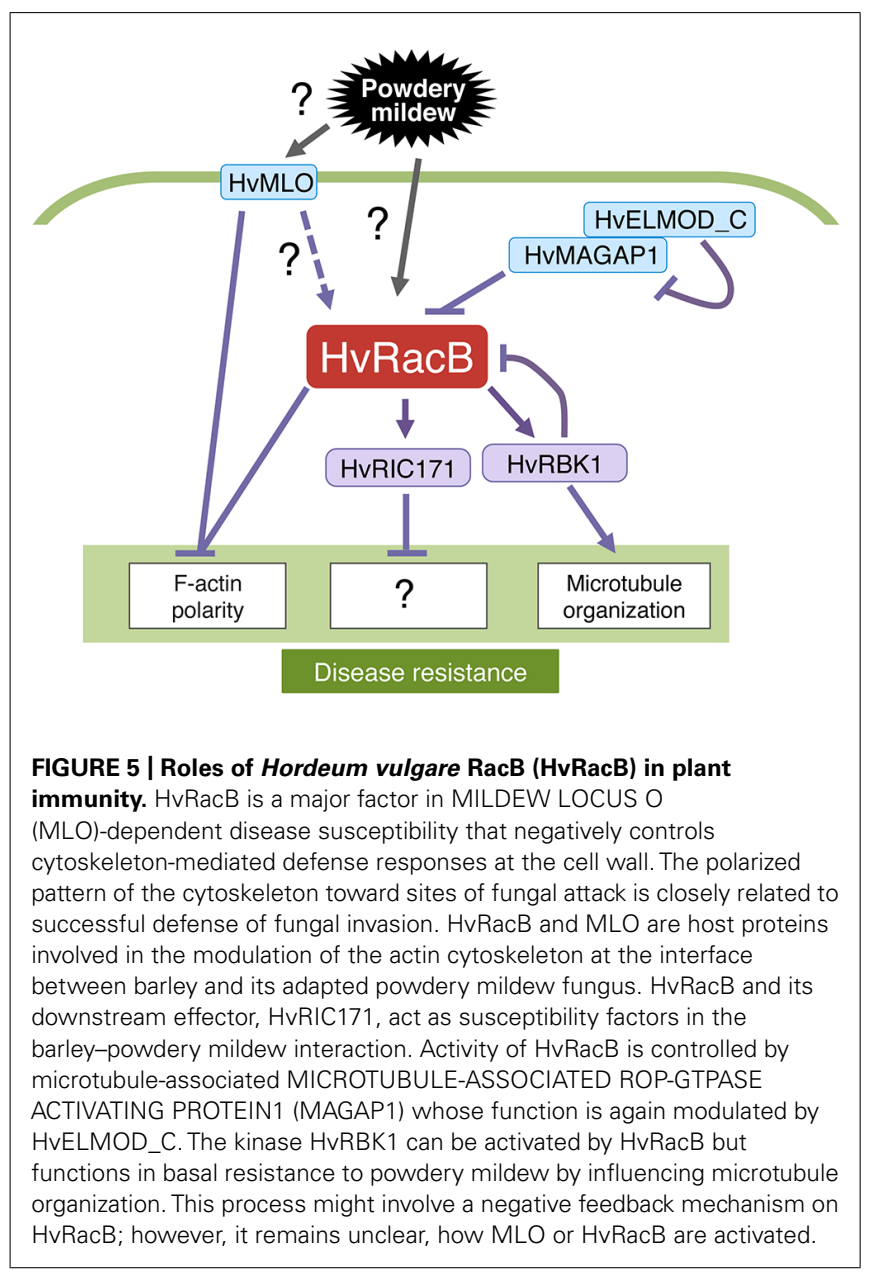

results suggest HvRacB and its downstream effector HvRIC171 act as susceptibility factors in the barley-powdery mildew interaction.

A barley MICROTUBULE-ASSOCIATED ROP-GTPASE ACTIVATING PROTEIN1 (MAGAP1) also interacts with HvRacB (Figure 5; Hoefle etal., 2011). MAGAP1 is localized along cortical microtubules and is recruited by activated HvRacB to the cell periphery. During fungal attack, MAGAP1-labeled microtubules form a polarized network at sites of successful defense. By contrast, microtubules loosen at the invasion sites where the fungus succeeds. A MAGAP1 mutant lacking GAP activity demonstrated that MAGAP1 is a limiting factor for susceptibility to penetration by $B g h$. Moreover, MAGAP1 regulates the polarized distribution of cortical microtubules toward sites of infections. This finding supports the hypothesis that HvRacB and MAGAP1 act antagonistically in cytoskeleton organization during fungal entry (Hoefle et al., 2011). Engulfment and Motility (ELMO) proteins participate in the regulation of small GTPase activity in eukaryotic organisms. The barley ELMO-Domain Containing Protein (HvELMOD_C) is partially associated with microtubule-associated MAGAP1 (Hoefle and Huckelhoven, 2014). The expression of HvELMOD_C compromises the resistance-inducing effect of HvMAGAP1 to Bgh when simultaneously expressed with HvMAGAP1. Thus, it is likely that 
HvELMOD_C works as a new modulator of Rac/Rop signaling in barley. Furthermore, CA-HvRacB interacts with a ROP-binding protein kinase (HvRBK1; Huesmann et al., 2012). The kinase activity of HvRBK1 is enhanced by the addition of CA-HvRacB in vitro. $H v R B K 1$ RNAi enhanced the penetration of barley epidermal cells by $B g h$ and lowered the stability of cortical microtubules. Thus, HvRBK1 might function in basal resistance to powdery mildew by influencing microtubule organization or by a negative feedback on the susceptibility factor HvRacB. Further studies are necessary to elucidate how HvRacB orchestrates the two cytoskeletons, actin, and microtubules, during powdery mildew infection.

\section{Rho PROTEINS AND ETI IN ANIMALS}

Recent studies have revealed that the involvement of the Rho family of proteins in NLR-dependent ETI signaling is conserved between plants and animals (Figure 4; Stuart and Boyer, 2013). In fact, co-immunoprecipitation assays with Racl show that Homo sapiens Racl (HsRac1) associates with NLR proteins NB oligomerization domain-containing protein 1 (NOD1) as well as NOD2, and OsRac1 directly interacts with the NLR protein Pit in rice (Legrand-Poels et al., 2007; Mayor et al., 2007; Kawano etal., 2010a; Keestra etal., 2013). Drosophila Toll, and the homologous Toll-like receptors in animals are PRRs that act as immune receptors of microbes. Both Salmonella typhimurium and Shigella flexneri are pathogens that invade host animal cells using a type III secretion system that is able to inject their effectors into host cells. Salmonella stimulates these responses by delivering through its type III secretion system the bacterial effector proteins SopE, SopE2, and SopB, which in a redundant fashion stimulate Rho family GTPases leading to the activation of MAPK and signaling by the transcription factor Nuclear factor- $\kappa \mathrm{B}$ (NF- $\kappa \mathrm{B}$; Bruno et al., 2009). The NLR protein NOD1 senses cytosolic microbial products by monitoring the activation state of Rac/Rop family proteins including HsRac1, HsRhoA HsCdc42 (Keestra etal., 2013). Activation of HsRacl and HsCdc42 by bacterial delivery or ectopic expression of SopE, an effector protein of Salmonella, triggered NOD1 signaling. In concert with a downstream kinase of NOD1, Receptor-interacting protein 2 (RIP2) mediated the induction of NF- $\mathrm{B}$-dependent inflammatory responses. Similarly, activation of the NOD1 signaling pathway by peptidoglycan (PGN) required HsRacl activity. CAHsRac1, HsCdc42, and HsRhoA activated the NOD1 signaling pathway.

Furthermore, GEF-H1 is a central component of pathogen recognition by NOD1 in animals (Figure 4; Fukazawa et al., 2008). Together, GEF-H1 and NOD1 not only detect the presence of PGN-derived muropeptides but also signal in response to Shigella effectors in the cytoplasm. GEF-H1 is recruited into bacterial invasion sites of S. flexneri, and subsequent Mus musculus RhoA (MmRhoA) small GTPase activation is required for cell invasion. In addition, GEF-H1 is requisite for the activation of NF-кB-dependent gene expression during Shigella invasion. GEF-H1 interacts with NOD1 and is required for NF-кB activation in response to PGN degradation products. Importantly, the Shigella effectors IpgB2 and OspB activate NF-кB by a mechanism

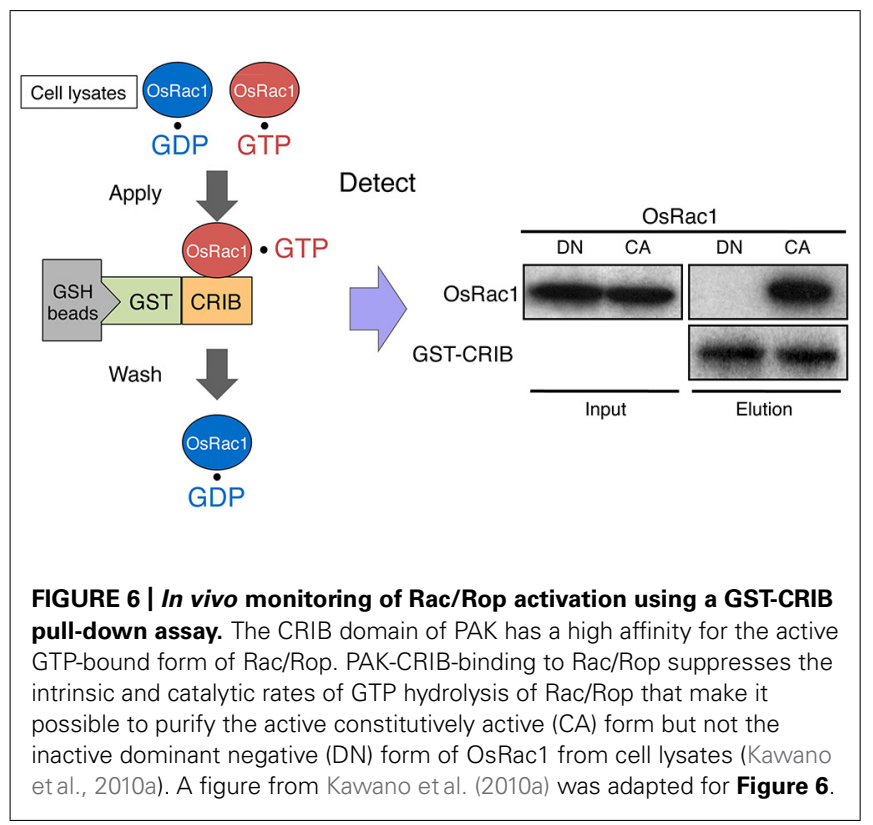

that depends on both NOD1 and GEF-H1 and requires Rhoassociated kinase (Rho-kinase) activation. GEF-H1 is a central component in a detection system that directs NF- $\kappa \mathrm{B}$ activation in MmRhoA- and RIP2-dependent pathways initiated by the action of bacterial effectors and intracellular pathogen pattern recognition.

In animals, the host indirectly senses the pathogen by monitoring for cytotoxic necrotizing factor 1 (CNF1), an Escherichia coli-derived effector molecule (Boyer et al., 2011). CNF1 modifies Drosophila melanogaster Rac2 that then interacts with the innate immune adaptors Immune deficiency (IMD) and RIP1RIP2 in fly and animal cells, respectively, to trigger an immune response.

Previous genetic studies have demonstrated that plant $\mathrm{R}$ protein functions are determined by multiple (co)chaperone proteins including SGT1, RAR1, and HSP90 (Shirasu, 2009). Interestingly, Homo sapiens SGT1 RNAi prevents multiple cellular responses associated with NOD1 activation, indicating that HsSGT1 positively regulates NOD1 activation (da Silva Correia et al., 2007). Knockdown of MmSGT1 or chemical inhibition of MmHSP90 abrogates inflammasome activity, and inhibition of MmHSP90 blocks NOD2-mediated activation of NF-kB and reduces NLR protein NALP3-mediated gout-like inflammation in mice (Mayor et al., 2007). The components of signal transduction in ETI are conserved among species. We noted that, in animals, NOD1 monitors the activation state of the Rho family proteins that are targeted by virulence effectors produced by pathogenic microbes (Figure 4). The mechanism for recognizing NLR proteins in animals shows striking similarities with the NLR protein recognition mechanism in plants through host proteins, called guardees, such as Arabidopsis RIN4 (Jones and Dangl, 2006). In contrast, OsRac1 appears to act downstream of the NLR proteins. At present, the precise differences in the roles of Rho family proteins in ETI signaling between animals and plants are largely unknown. Further studies are necessary to clarify these relationships. 


\section{IN VIVO MONITORING OF Rac/Rop ACTIVATION}

Given that Rac/Rop is a master regulator controlling plant immunity, monitoring its activation within plant cells is believed to be the next key step in understanding plant immunity. Traditionally, small GTPase activities are measured using in vivo labeling of cells with of inorganic $\left[{ }^{32} \mathrm{P}\right]$ phosphate followed by isolation of the GTPase and thin-layer chromatography of bound guanine nucleotides. This method provides quantitative data for GDP and GTP levels on small GTPases but is a time-consuming procedure that requires large amounts of radioisotopes. Currently, we are able to use two alternative non-radioactive techniques, a PAK-CRIB pull-down assay and a Raichu-Förster resonance energy transfer (FRET) sensor, to monitor the in vivo activation of Rac/Rop (Figures 6 and 7; Sander etal., 1998; Mochizuki etal., 2001; Tao etal., 2002; Kawano et al., 2010a). These methods exploit the selective interaction of the CRIB of the Rac-effector PAK1 in animals. Since the CRIB-domain of PAK has a high affinity for the active GTP-bound form of Rac/Rop and PAK-CRIB binding results in a significantly reduced intrinsic GTPase activity of Rac/Rop, these factors result in an ideal tool for affinity purification of active GTP-bound forms of Rac/Rop from crude cell lysates. Recombinant GST-tagged PAK-CRIB protein is currently available from several manufacturers. GST-tagged PAK-CRIB allows one to "pull-down" the PAK-CRIB/GTP-Rac/Rop complex with glutathione affinity beads (Figure 6). Therefore, the assay provides a simple means of quantifying Rac/Rop activation in cells. The amount of activated Rac is determined by immunoblotting. This approach has greatly accelerated and, thus, simplified the semi-quantitative measurement of Rac activity in plants and animals (Sander etal., 1998; Tao etal., 2002; Xu etal., 2010).

We have established a FRET probe, Ras and interacting protein chimeric unit (Raichu)-OsRac1, for monitoring the activation of OsRac1 in living cells (Figure 7; Mochizuki et al., 2001; Kawano et al., 2010a). Raichu was originally developed to study the activation of various small GTPases, including Rac1, in mammalian cells (Mochizuki etal., 2001; Itoh et al., 2002). Raichu and its variants are well-established tools for monitoring the activation of small GTPases among species. Raichu-OsRac1 is composed of OsRac1, the CRIB domain of PAK1, which binds specifically to the GTP-bound form of OsRac1, and the FRET donor (YFP) and the FRET acceptor (CFP). Intramolecular binding of active GTP.OsRac1 to CRIB brings CFP closer to YFP, enabling FRET from CFP to YFP to occur. The resulting YFP fluorescence provides an estimate of the activation state of OsRacl in vivo, with low and high ratios of YFP/CFP fluorescence corresponding to low and high levels of OsRacl activation, respectively. Using Raichu-OsRac1, we identified the R protein Pit that activated OsRacl on the plasma membrane (Kawano et al., 2010a) and observed that OsRacl was activated within 3 min after sensing chitin, a cell wall component of the rice blast fungus (Figure 7; Akamatsu etal., 2013). The Raichu system is powerful tool for monitoring activation states of small GTPase, thus, we hope that this system becomes widely used in the field of plant biology for understanding the spatio-temporal characteristics of small GTPase activation.

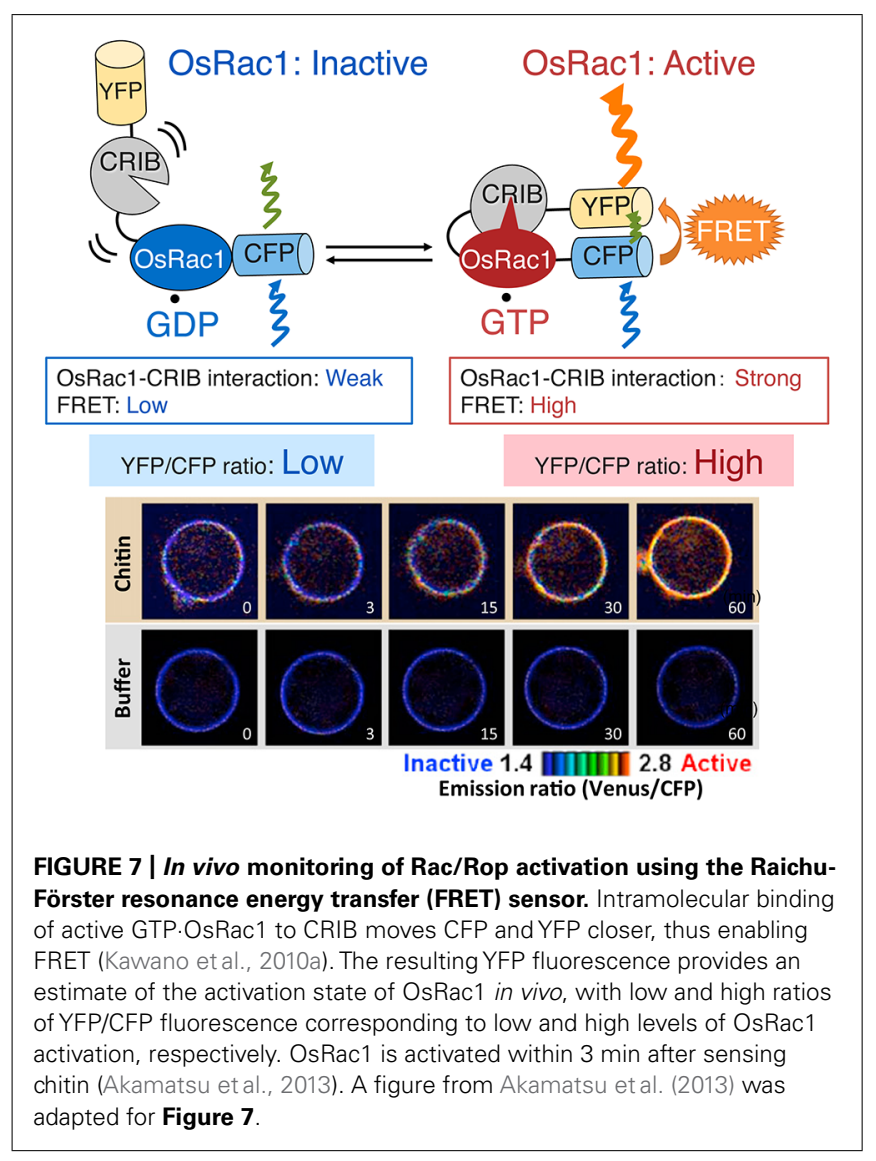

\section{CONCLUDING REMARKS}

The study of Rac/Rop family GTPase-dependent plant immunity is a rapidly expanding field. Recently, progress has been made in elucidating the defense mechanisms of rice OsRacl to rice blast fungus (Figures 3 and $\mathbf{4}$ ) and barley HvRacB to powdery mildew (Figure 5). The detection of pathogen-derived effectors using NLR proteins between plants and animals is highly conserved (Figure 4). There is a high likelihood that the NLR proteins arose from a primitive innate immune system, and it will be interesting to elucidate the evolutionary process. Currently, our knowledge of the downstream signaling components in ETI is limited. More biochemical and structural studies are required to understand the possible mechanisms in ETI.

\section{ACKNOWLEDGMENTS}

Because of limitations in the number of references for this review, we were unable to cite the work of many of our colleagues that have greatly contributed to this area of research. We thank Dr. Ralph Hueckelhoven, Technische Universitaet Muenchen, for critically reading this manuscript. We also thank the members of the Laboratory of Plant Molecular Genetics for invaluable comments and discussions. This research was supported by JSPS KAKENHI Grant Number 2377044, 24113515, 26113712, 26450055 to Yoji Kawano, by Takeda Science Foundation to Yoji Kawano, and by Inamori Foundation and Ichiro Kanehara Foundation for Promotion of Medical Sciences, Research Promotion Program of Ritsumeikan University, and Medical Care to Takako Kaneko-Kawano. 


\section{REFERENCES}

Akamatsu, A., Wong, H., Fujiwara, M., Okuda, J., Nishide, K., Uno, K., et al. (2013). An OsCEBiP/OsCERK1-OsRacGEF1-OsRacl module is an essential component of chitin-induced rice immunity. Cell Host Microbe 13, 465-476. doi: 10.1016/j.chom.2013.03.007

Asai, T., Tena, G., Plotnikova, J., Willmann, M. R., Chiu, W. L., Gomez-Gomez, L., et al. (2002). MAP kinase signalling cascade in Arabidopsis innate immunity. Nature 415, 977-983. doi: 10.1038/415977a

Baxter-Burrell, A., Yang, Z., Springer, P. S., and Bailey-Serres, J. (2002). RopGAP4dependent Rop GTPase rheostat control of Arabidopsis oxygen deprivation tolerance. Science 296, 2026-2028. doi: 10.1126/science.1071505

Berken, A. (2006). ROPs in the spotlight of plant signal transduction. Cell. Mol. Life Sci. 63, 2446-2459. doi: 10.1007/s00018-006-6197-1

Berken, A., Thomas, C., and Wittinghofer, A. (2005). A new family of RhoGEFs activates the Rop molecular switch in plants. Nature 436, 1176-1180. doi: 10.1038 /nature 03883

Boerjan, W., Ralph, J., and Baucher, M. (2003). Lignin biosynthesis. Annu. Rev. Plant Biol. 54, 519-546. doi: 10.1146/annurev.arplant.54.031902.134938

Bos, J. L., Rehmann, H., and Wittinghofer, A. (2007). GEFs and GAPs: critical elements in the control of small G proteins. Cell 129, 865-877. doi: 10.1016/j.cell.2007.05.018

Boyer, L., Magoc, L., Dejardin, S., Cappillino, M., Paquette, N., Hinault, C., et al. (2011). Pathogen-derived effectors trigger protective immunity via activation of the Rac2 enzyme and the IMD or Rip kinase signaling pathway. Immunity 35, 536-549. doi: 10.1016/j.immuni.2011.08.015

Bruno, V. M., Hannemann, S., Lara-Tejero, M., Flavell, R. A., Kleinstein, S. H., and Galan, J. E. (2009). Salmonella typhimurium type III secretion effectors stimulate innate immune responses in cultured epithelial cells. PLoS Pathog. 5:e1000538. doi: 10.1371/journal.ppat.1000538

Césari, S., Kanzaki, H., Fujiwara, T., Bernoux, M., Chalvon, V., Kawano, Y., et al. (2014). The NB-LRR proteins RGA4 and RGA5 interact functionally and physically to confer disease resistance. $E M B O J .33,1941-1959$. doi: 10.15252/embj.201487923

Cesari, S., Thilliez, G., Ribot, C., Chalvon, V., Michel, C., Jauneau, A., et al. (2013). The rice resistance protein pair RGA4/RGA5 recognizes the Magnaporthe oryzae effectors AVR-Pia and AVR1-CO39 by direct binding. Plant Cell 25, 1463-1481. doi: $10.1105 /$ tpc.112.107201

Chen, J. G., Ullah, H., Temple, B., Liang, J., Guo, J., Alonso, J. M., et al. (2006). RACK1 mediates multiple hormone responsiveness and developmental processes in Arabidopsis. J. Exp. Bot. 57, 2697-2708. doi: 10.1093/jxb/erl035

Chen, L., Hamada, S., Fujiwara, M., Zhu, T., Thao, N. P., Wong, H. L., et al. (2010a). The Hop/Sti1-Hsp90 chaperone complex facilitates the maturation and transport of a PAMP receptor in rice innate immunity. Cell Host Microbe 7, 185-196. doi: 10.1016/j.chom.2010.02.008

Chen, L., Shiotani, K., Togashi, T., Miki, D., Aoyama, M., Wong, H. L., et al. (2010b). Analysis of the Rac/Rop small GTPase family in rice: expression, subcellular localization and role in disease resistance. Plant Cell Physiol. 51, 585-595. doi: $10.1093 / \mathrm{pcp} / \mathrm{pcq} 024$

da Silva Correia, J., Miranda, Y., Leonard, N., and Ulevitch, R. (2007). SGT1 is essential for Nod1 activation. Proc. Natl. Acad. Sci. U.S.A. 104, 6764-6769. doi: 10.1073/pnas.0610926104

Dodds, P. N., and Rathjen, J. P. (2010). Plant immunity: towards an integrated view of plant-pathogen interactions. Nat. Rev. Genet. 11, 539-548. doi: $10.1038 / \mathrm{nrg} 2812$

Fukazawa, A., Alonso, C., Kurachi, K., Gupta, S., Lesser, C. F., McCormick, B. A., et al. (2008). GEF-H1 mediated control of NOD1 dependent NF-kB activation by Shigella effectors. PLoS Pathog. 4:e1000228. doi: 10.1371/journal.ppat.1000228

Gust, A. A., Willmann, R., Desaki, Y., Grabherr, H. M., and Nurnberger, T. (2012). Plant LysM proteins: modules mediating symbiosis and immunity. Trends Plant Sci. 17, 495-502. doi: 10.1016/j.tplants.2012.04.003

Hoefle, C., and Huckelhoven, R. (2014). A barley Engulfment and Motility domain containing protein modulates Rho GTPase activating protein HvMAGAP1 function in the barley powdery mildew interaction. Plant Mol. Biol. 84, 469-478. doi: 10.1007/s11103-013-0145-x

Hoefle, C., Huesmann, C., Schultheiss, H., Bornke, F., Hensel, G., Kumlehn, J., et al. (2011). A barley ROP GTPase ACTIVATING PROTEIN associates with microtubules and regulates entry of the barley powdery mildew fungus into leaf epidermal cells. Plant Cell 23, 2422-2439. doi: 10.1105/tpc.110.082131
Huesmann, C., Reiner, T., Hoefle, C., Preuss, J., Jurca, M. E., Domoki, M., et al. (2012). Barley ROP binding kinasel is involved in microtubule organization and in basal penetration resistance to the barley powdery mildew fungus. Plant Physiol. 159, 311-320. doi: 10.1104/pp.111.191940

Itoh, R. E., Kurokawa, K., Ohba, Y., Yoshizaki, H., Mochizuki, N., and Matsuda, M. (2002). Activation of rac and cdc42 video imaged by fluorescent resonance energy transfer-based single-molecule probes in the membrane of living cells. Mol. Cell. Biol. 22, 6582-6591. doi: 10.1128/MCB.22.18.6582-6591.2002

Iwata, M., Umemura, K., Teraoka, T., Usami, H., Fujisawa, Y., and Iwasaki, Y. (2003). Role of the $\alpha$ subunit of heterotrimeric G-protein in probenazoleinducing defense signaling in rice. J. Gen. Plant Pathol. 69, 83-86. doi: 10.1007/s10327-002-0008-9

Jones, J. D., and Dangl, J. L. (2006). The plant immune system. Nature 444, 323-329. doi: 10.1038/nature05286

Jung, Y. H., Agrawal, G. K., Rakwal, R., Kim, J. A., Lee, M. O., Choi, P. G., et al. (2006). Functional characterization of OsRacB GTPase - a potentially negative regulator of basal disease resistance in rice. Plant Physiol. Biochem. 44, 68-77. doi: 10.1016/j.plaphy.2005.12.001

Kaku, H., Nishizawa, Y., Ishii-Minami, N., Akimoto-Tomiyama, C., Dohmae, N., Takio, K., et al. (2006). Plant cells recognize chitin fragments for defense signaling through a plasma membrane receptor. Proc. Natl. Acad. Sci. U.S.A. 103, 1108611091. doi: 10.1073/pnas.0508882103

Kaothien, P., Ok, S. H., Shuai, B., Wengier, D., Cotter, R., Kelley, D., et al. (2005). Kinase partner protein interacts with the LePRK1 and LePRK2 receptor kinases and plays a role in polarized pollen tube growth. Plant J. 42, 492-503. doi: 10.1111/j.1365-313X.2005.02388.x

Kawano, Y., Akamatsu, A., Hayashi, K., Housen, Y., Okuda, J., Yao, A., et al. (2010a). Activation of a Rac GTPase by the NLR family disease resistance protein Pit plays a critical role in rice innate immunity. Cell Host Microbe 7, 362-375. doi: 10.1016/j.chom.2010.04.010

Kawano, Y., Chen, L., and Shimamoto, K. (2010b). The function of Rac small GTPase and associated proteins in rice innate immunity. Rice 3, 112-121. doi: 10.1007/s12284-010-9049-4

Kawano, Y., Fujiwara, T., Yao, A., Housen, Y., Hayashi, K., and Shimamoto, K. (2014). Palmitoylation-dependent membrane localization of the rice $\mathrm{R}$ protein Pit is critical for the activation of the small GTPase OsRac1. J. Biol. Chem. 289, 19079-19088. doi: $10.1074 /$ jbc.M114.569756

Kawano, Y., and Shimamoto, K. (2013). Early signaling network in rice PRR- and R-mediated immunity. Curr. Opin. Plant Biol. 16, 496-504. doi: 10.1016/j.pbi.2013.07.004

Kawasaki, T., Henmi, K., Ono, E., Hatakeyama, S., Iwano, M., Satoh, H., et al. (1999). The small GTP-binding protein Rac is a regulator of cell death in plants. Proc. Natl. Acad. Sci. U.S.A. 96, 10922-10926. doi: 10.1073/pnas.96.19.10922

Kawasaki, T., Koita, H., Nakatsubo, T., Hasegawa, K., Wakabayashi, K., Takahashi, H., et al. (2006). Cinnamoyl-CoA reductase, a key enzyme in lignin biosynthesis, is an effector of small GTPase Rac in defense signaling in rice. Proc. Natl. Acad. Sci. U.S.A. 103, 230-235. doi: 10.1073/pnas.0509875103

Keestra, A. M., Winter, M. G., Auburger, J. J., Frassle, S. P., Xavier, M. N., Winter, S. E., et al. (2013). Manipulation of small Rho GTPases is a pathogeninduced process detected by NOD1. Nature 496, 233-237. doi: 10.1038/ nature 12025

Kim, S. H., Oikawa, T., Kyozuka, J., Wong, H. L., Umemura, K., Kishi-Kaboshi, M., et al. (2012). The bHLH Rac immunityl (RAI1) is activated by OsRacl via OsMAPK3 and OsMAPK6 in rice immunity. Plant Cell Physiol. 53, 740-754. doi: $10.1093 / \mathrm{pcp} / \mathrm{pcs} 033$

Kishi-Kaboshi, M., Okada, K., Kurimoto, L., Murakami, S., Umezawa, T., Shibuya, N., et al. (2010). A rice fungal MAMP-responsive MAPK cascade regulates metabolic flow to antimicrobial metabolite synthesis. Plant J. 63, 599-612. doi: 10.1111/j.1365-313X.2010.04264.x

Kosami, K. I., Ohki, I., Nagano, M., Furuita, K., Sugiki, T., Kawano, Y., et al. (2014). The crystal structure of the plant small GTPase OsRac1 reveals its mode of binding to NADPH oxidase. J. Biol. Chem. doi: 10.1074/jbc.M114.603282 [Epub ahead of print].

Lavy, M., Bracha-Drori, K., Sternberg, H., and Yalovsky, S. (2002). A cell-specific, prenylation-independent mechanism regulates targeting of type II RACs. Plant Cell 14, 2431-2450. doi: 10.1105/tpc.005561

Legrand-Poels, S., Kustermans, G., Bex, F., Kremmer, E., Kufer, T. A., and Piette, J. (2007). Modulation of Nod2-dependent NF-kappaB signaling by the actin cytoskeleton. J. Cell Sci. 120, 1299-1310. doi: 10.1242/jcs.03424 
Li, H., Wu, G., Ware, D., Davis, K. R., and Yang, Z. (1998). Arabidopsis Rho-related GTPases: differential gene expression in pollen and polar localization in fission yeast. Plant Physiol. 118, 407-417. doi: 10.1104/pp.118.2.407

Lieberherr, D., Thao, N. P., Nakashima, A., Umemura, K., Kawasaki, T., and Shimamoto, K. (2005). A sphingolipid elicitor-inducible mitogen-activated protein kinase is regulated by the small GTPase OsRacl and heterotrimeric G-protein in rice. Plant Physiol. 138, 1644-1652. doi: 10.1104/pp.104.057414

Lukasik, E., and Takken, F. L. (2009). STANDing strong, resistance proteins instigators of plant defence. Curr. Opin. Plant Biol. 12, 427-436. doi: 10.1016/j.pbi.2009.03.001

Mayor, A., Martinon, F., De Smedt, T., Petrilli, V., and Tschopp, J. (2007). A crucial function of SGT1 and HSP90 in inflammasome activity links mammalian and plant innate immune responses. Nat. Immunol. 8, 497-503. doi: 10.1038/ ni1459

McCahill, A., Warwicker, J., Bolger, G. B., Houslay, M. D., and Yarwood, S. J. (2002). The RACK1 scaffold protein: a dynamic cog in cell response mechanisms. Mol Pharmacol. 62, 1261-1273. doi: 10.1124/mol.62.6.1261

Miki, D., Itoh, R., and Shimamoto, K. (2005). RNA silencing of single and multiple members in a gene family of rice. Plant Physiol. 138, 1903-1913. doi: 10.1104/pp.105.063933

Mochizuki, N., Yamashita, S., Kurokawa, K., Ohba, Y., Nagai, T., Miyawaki, A., et al. (2001). Spatio-temporal images of growth-factor-induced activation of Ras and Rap1. Nature 411, 1065-1068. doi: 10.1038/35082594

Moeder, W., Yoshioka, K., and Klessig, D. F. (2005). Involvement of the small GTPase Rac in the defense responses of tobacco to pathogens. Mol. Plant Microbe Interact. 18, 116-124. doi: 10.1094/MPMI-18-0116

Monaghan, J., and Zipfel, C. (2012). Plant pattern recognition receptor complexes at the plasma membrane. Curr. Opin. Plant Biol. 15, 349-357. doi: 10.1016/j.pbi.2012.05.006

Nakashima, A., Chen, L., Thao, N. P., Fujiwara, M., Wong, H. L., Kuwano, M., et al. (2008). RACK1 functions in rice innate immunity by interacting with the Racl immune complex. Plant Cell 20, 2265-2279. doi: 10.1105/tpc.107.054395

Oda, T., Hashimoto, H., Kuwabara, N., Akashi, S., Hayashi, K., Kojima, C., et al. (2010). Structure of the N-terminal regulatory domain of a plant NADPH oxidase and its functional implications. J. Biol. Chem. 285, 1435-1445. doi: 10.1074/jbc.M109.058909

Okuyama, Y., Kanzaki, H., Abe, A., Yoshida, K., Tamiru, M., Saitoh, H., et al. (2011). A multifaceted genomics approach allows the isolation of the rice Piablast resistance gene consisting of two adjacent NBS-LRR protein genes. Plant J. 66, 467-479. doi: 10.1111/j.1365-313X.2011.04502.x

Ono, E., Wong, H. L., Kawasaki, T., Hasegawa, M., Kodama, O., and Shimamoto, K. (2001). Essential role of the small GTPase Rac in disease resistance of rice. Proc. Natl. Acad. Sci. U.S.A. 98, 759-764. doi: 10.1073/pnas.98.2.759

Opalski, K. S., Schultheiss, H., Kogel, K. H., and Huckelhoven, R. (2005). The receptor-like MLO protein and the RAC/ROP family G-protein RACB modulate actin reorganization in barley attacked by the biotrophic powdery mildew fungus Blumeria graminis f.sp. hordei. Plant J. 41, 291-303. doi: 10.1111/j.1365-313X.2004.02292.x

Paduch, M., Jelen, F., and Otlewski, J. (2001). Structure of small G proteins and their regulators. Acta Biochim. Pol. 48, 829-850.

Park, J., Gu, Y., Lee, Y., Yang, Z., and Lee, Y. (2004). Phosphatidic acid induces leaf cell death in Arabidopsis by activating the Rho-related small G protein GTPasemediated pathway of reactive oxygen species generation. Plant Physiol. 134, 129 136. doi: 10.1104/pp.103.031393

Pathuri, I. P., Zellerhoff, N., Schaffrath, U., Hensel, G., Kumlehn, J., Kogel, K. H., et al. (2008). Constitutively activated barley ROPs modulate epidermal cell size, defense reactions and interactions with fungal leaf pathogens. Plant Cell Rep. 27, 1877-1887. doi: 10.1007/s00299-008-0607-9

Poraty-Gavra, L., Zimmermann, P., Haigis, S., Bednarek, P., Hazak, O., Stelmakh, O. R., et al. (2013). The Arabidopsis Rho of plants GTPase AtROP6 functions in developmental and pathogen response pathways. Plant Physiol. 161, 1172-1188. doi: 10.1104/pp.112.213165

Potikha, T. S., Collins, C. C., Johnson, D. I., Delmer, D. P., and Levine, A. (1999). The involvement of hydrogen peroxide in the differentiation of secondary walls in cotton fibers. Plant Physiol. 119, 849-858. doi: 10.1104/pp.119.3.849

Rodriguez, M. C., Petersen, M., and Mundy, J. (2010). Mitogen-activated protein kinase signaling in plants. Annu. Rev. Plant Biol. 61, 621-649. doi: 10.1146/annurev-arplant-042809-112252
Samajova, O., Komis, G., and Samaj, J. (2013). Emerging topics in the cell biology of mitogen-activated protein kinases. Trends Plant Sci. 18, 140-148. doi: 10.1016/j.tplants.2012.11.004

Sander, E. E., van Delft, S., ten Klooster, J. P., Reid, T., van der Kammen, R. A., Michiels, F., et al. (1998). Matrix-dependent Tiam1/Rac signaling in epithelial cells promotes either cell-cell adhesion or cell migration and is regulated by phosphatidylinositol 3-kinase. J. Cell Biol. 143, 1385-1398. doi: 10.1083/jcb.143.5.1385

Schultheiss, H., Dechert, C., Kogel, K. H., and Huckelhoven, R. (2002). A small GTP-binding host protein is required for entry of powdery mildew fungus into epidermal cells of barley. Plant Physiol. 128, 1447-1454. doi: 10.1104/pp. 010805

Schultheiss, H., Dechert, C., Kogel, K. H., and Huckelhoven, R. (2003). Functional analysis of barley RAC/ROP G-protein family members in susceptibility to the powdery mildew fungus. Plant J. 36, 589-601. doi: 10.1046/j.1365313X.2003.01905.x

Schultheiss, H., Hensel, G., Imani, J., Broeders, S., Sonnewald, U., Kogel, K. H., et al. (2005). Ectopic expression of constitutively activated RACB in barley enhances susceptibility to powdery mildew and abiotic stress. Plant Physiol. 139, 353-362. doi: 10.1104/pp.105.066613

Schultheiss, H., Preuss, J., Pircher, T., Eichmann, R., and Huckelhoven, R. (2008). Barley RIC171 interacts with RACB in planta and supports entry of the powdery mildew fungus. Cell Microbiol. 10, 1815-1826. doi: 10.1111/j.14625822.2008.01167.x

Shimizu, T., Nakano, T., Takamizawa, D., Desaki, Y., Ishii-Minami, N., Nishizawa, Y., et al. (2010). Two LysM receptor molecules, CEBiP and OsCERK1, cooperatively regulate chitin elicitor signaling in rice. Plant J. 64, 204-214. doi: 10.1111/j.1365313X.2010.04324.x

Shinohara, M., Terada, Y., Iwamatsu, A., Shinohara, A., Mochizuki, N., Higuchi, M., et al. (2002). SWAP-70 is a guanine-nucleotide-exchange factor that mediates signalling of membrane ruffling. Nature 416, 759-763. doi: 10.1038/416759a

Shinya, T., Motoyama, N., Ikeda, A., Wada, M., Kamiya, K., Hayafune, M., et al. (2012). Functional characterization of CEBiP and CERK1 homologs in Arabidopsis and rice reveals the presence of different chitin receptor systems in plants. Plant Cell Physiol. 53, 1696-1706. doi: 10.1093/pcp/pcs113

Shirasu, K. (2009). The HSP90-SGT1 chaperone complex for NLR immune sensors. Annu. Rev. Plant Biol. 60, 139-164. doi: 10.1146/annurev.arplant.59.032607. 092906

Shiu, S. H., Karlowski, W. M., Pan, R., Tzeng, Y. H., Mayer, K. F., and Li, W. H. (2004). Comparative analysis of the receptor-like kinase family in Arabidopsis and rice. Plant Cell 16, 1220-1234. doi: 10.1105/tpc.020834

Stuart, L. M., and Boyer, L. (2013). RhoGTPases - NODes for effector-triggered immunity in animals. Cell Res. 23, 980-981. doi: 10.1038/cr.2013.68

Suharsono, U., Fujisawa, Y., Kawasaki, T., Iwasaki, Y., Satoh, H., and Shimamoto, K. (2002). The heterotrimeric G protein alpha subunit acts upstream of the small GTPase Rac in disease resistance of rice. Proc. Natl. Acad. Sci. U.S.A. 99, 13307-13312. doi: 10.1073/pnas. 192244099

Tao, L. Z., Cheung, A. Y., and Wu, H. M. (2002). Plant Rac-like GTPases are activated by auxin and mediate auxin-responsive gene expression. Plant Cell 14, 2745-2760. doi: $10.1105 /$ tpc.006320

Thao, N. P., Chen, L., Nakashima, A., Hara, S., Umemura, K., Takahashi, A., et al. (2007). RAR1 and HSP90 form a complex with Rac/Rop GTPase and function in innate-immune responses in rice. Plant Cell 19, 4035-4045. doi: 10.1105/tpc.107.055517

Thomas, C., Fricke, I., Scrima, A., Berken, A., and Wittinghofer, A. (2007). Structural evidence for a common intermediate in small G protein-GEF reactions. Mol. Cell 25, 141-149. doi: 10.1016/j.molcel.2006.11.023

Torres, M. A., and Dangl, J. L. (2005). Functions of the respiratory burst oxidase in biotic interactions, abiotic stress and development. Curr. Opin. Plant Biol. 8, 397-403. doi: 10.1016/j.pbi.2005.05.014

Trotochaud, A. E., Hao, T., Wu, G., Yang, Z., and Clark, S. E. (1999). The CLAVATA1 receptor-like kinase requires CLAVATA3 for its assembly into a signaling complex that includes KAPP and a Rho-related protein. Plant Cell 11, 393-406. doi: 10.1105/tpc.11.3.393

van der Luit, A. H., Piatti, T., van Doorn, A., Musgrave, A., Felix, G., Boller, T., et al. (2000). Elicitation of suspension-cultured tomato cells triggers the formation of phosphatidic acid and diacylglycerol pyrophosphate. Plant Physiol. 123, 15071516. doi: 10.1104/pp.123.4.1507 
Wamaitha, M., Yamamoto, R., Wong, H., Kawasaki, T., Kawano, Y., and Shimamoto, K. (2012). OsRap2.6 transcription factor contributes to rice innate immunity through its interaction with Receptor for Activated Kinase-C 1 (RACK1). Rice 5:35. doi: 10.1186/1939-8433-5-35

Wang, Y., Gao, M., Li, Q., Wang, L., Wang, J., Jeon, J. S., et al. (2008). OsRAR1 and OsSGT1 physically interact and function in rice basal disease resistance. Mol Plant Microbe Interact. 21, 294-303. doi: 10.1094/MPMI-21-3-0294

Wennerberg, K., Rossman, K. L., and Der, C. J. (2005). The Ras superfamily at a glance. J. Cell Sci. 118, 843-846. doi: 10.1242/jcs.01660

Winge, P., Brembu, T., Kristensen, R., and Bones, A. M. (2000). Genetic structure and evolution of RAC-GTPases in Arabidopsis thaliana. Genetics 156, 1959-1971.

Wong, H. L., Pinontoan, R., Hayashi, K., Tabata, R., Yaeno, T., Hasegawa, K., et al. (2007). Regulation of rice NADPH oxidase by binding of Rac GTPase to its N-terminal extension. Plant Cell 19, 4022-4034. doi: 10.1105/tpc.107.055624

Wong, H. L., Sakamoto, T., Kawasaki, T., Umemura, K., and Shimamoto, K. (2004). Down-regulation of metallothionein, a reactive oxygen scavenger, by the small GTPase OsRacl in rice. Plant Physiol. 135, 1447-1456. doi: 10.1104/pp.103.036384

Xu, T., Wen, M., Nagawa, S., Fu, Y., Chen, J. G., Wu, M. J., et al. (2010). Cell surface- and rho GTPase-based auxin signaling controls cellular interdigitation in Arabidopsis. Cell 143, 99-110. doi: 10.1016/j.cell.2010.09.003

Yamaguchi, K., Imai, K., Akamatsu, A., Mihashi, M., Hayashi, N., Shimamoto, K., et al. (2012). SWAP70 functions as a Rac/Rop guanine nucleotide-exchange factor in rice. Plant J. 70, 389-397. doi: 10.1111/j.1365-313X.2011.04874.x

Yang, K. Y., Liu, Y., and Zhang, S. (2001). Activation of a mitogen-activated protein kinase pathway is involved in disease resistance in tobacco. Proc. Natl. Acad. Sci. U.S.A. 98, 741-746. doi: 10.1073/pnas.98.2.741
Young, S. A., Wang, X., and Leach, J. E. (1996). Changes in the plasma membrane distribution of rice phospholipase $\mathrm{D}$ during resistant interactions with Xanthomonas oryzae pv oryzae. Plant Cell 8, 1079-1090. doi: 10.1105/tpc.8. 6.1079

Zhang, Y., and McCormick, S. (2007). A distinct mechanism regulating a pollenspecific guanine nucleotide exchange factor for the small GTPase Rop in Arabidopsis thaliana. Proc. Natl. Acad. Sci. U.S.A. 104, 18830-18835. doi: 10.1073/pnas.0705874104

Conflict of Interest Statement: The authors declare that the research was conducted in the absence of any commercial or financial relationships that could be construed as a potential conflict of interest.

Received: 30 June 2014; paper pending published: 16 July 2014; accepted: 16 September 2014; published online: 14 October 2014.

Citation: Kawano Y, Kaneko-Kawano T and Shimamoto K (2014) Rho family GTPase-dependent immunity in plants and animals. Front. Plant Sci. 5:522. doi: $10.3389 / \mathrm{fpls} .2014 .00522$

This article was submitted to Plant Traffic and Transport, a section of the journal Frontiers in Plant Science.

Copyright (c) 2014 Kawano, Kaneko-Kawano and Shimamoto. This is an open-access article distributed under the terms of the Creative Commons Attribution License (CC BY). The use, distribution or reproduction in other forums is permitted, provided the original author(s) or licensor are credited and that the original publication in this journal is cited, in accordance with accepted academic practice. No use, distribution or reproduction is permitted which does not comply with these terms. 